\title{
Versatile Approach to the Spin Dynamics in Correlated Electron Systems
}

\author{
Malte Behrmann, ${ }^{1}$ Alexander I. Lichtenstein, ${ }^{1}$ Mikhail I. Katsnelson, ${ }^{2}$ and Frank Lechermann ${ }^{1,3}$ \\ ${ }^{1}$ I. Institut für Theoretische Physik, Universität Hamburg, D-20355 Hamburg, Germany \\ ${ }^{2}$ Radboud University Nijmegen, Institute for Molecules and Materials, NL-6525 AJ Nijmegen, The Netherlands \\ ${ }^{3}$ Institut für Keramische Hochleistungswerkstoffe, \\ Technische Universität Hamburg-Harburg, D-21073 Hamburg, Germany
}

\begin{abstract}
Time-dependent spin phenomena in condensed matter are most often either described in the weakly correlated limit of metallic Stoner/Slater-like magnetism via band theory or in the strongly correlated limit of Heisenberg-like interacting spins in an insulator. However many experimental studies, e.g. of (de)magnetization processes, focus on itinerant local-moment materials such as transition metals and various of their compounds. We here present a general theoretical framework that is capable of addressing correlated spin dynamics, also in the presence of a vanishing charge gap. A real-space implementation of the time-dependent rotational-invariant slave boson methodology allows to treat non-equilibrium spins numerically fast and efficiently beyond linear response as well as beyond the band-theoretical or Heisenberg limit.
\end{abstract}

\section{INTRODUCTION}

Non-equilibrium physics in challenging condensed matter electron systems, gained enormous attention in recent times. Highlight studies include e.g. driven (de)magnetization in transition-metal ${ }^{1}$ or rare-earth $^{2}$ systems, light-induced superconductivity ${ }^{3}$ or melting of charge-density waves ${ }^{4 / 5}$ Introducing explicit time dependence within interacting quantum materials could allow for a dynamic stabilization of equilibrium metastable states. Furthermore new pathways to novel exotic states of matter may thereby envisioned.

Dynamic magnetism is a key research focus in this respect, since it not only continues the history of longstanding studies of an ubiquitous solid-state phenomenon. There is also always the chance for groundbreaking technological applications. While experimental progress has been fast and investigations nowadays deal with a wide range of materials, the theoretical description struggles to keep up. So far only two limiting regimes are reasonably well accessible by theoretical means. First the metallic band-magnetism limit without the notion of localmoment physics, and second the insulating pure-spin limit where charge degrees of freedom are gapped. For the former case there are several modeling ideas within band theory dealing with itinerant Stoner physics out of equilibrium. Elliot-Yaffet(-like) theory ${ }^{6 / 7}$ e.g. is a widely utilized $\frac{891}{}$ theoretical framework to address metallic demagnetization experiments. Model Hamiltonians of Heisenberg kind are on the contrary often applied to the interacting problem of time-dependent localized lattice spins. ${ }^{10}$ Yet most concrete experimental studies focus on itinerant systems with coexisting local moments and/or involve some charge fluctuations in the dynamicprobing protocol. In fact, it is agreed that the interplay of band theory and electron correlation is at the heart of dynamic materials magnetism. $\frac{11}{13}$ Hence theory should be ready to tackle the generic problem of non-equilibrium magnetic phenomena aside from the pure-band and -spin limits.
Time-dependent (TD) correlation phenomena are describable in a close-to-exact numerical manner within TD density-matrix-renormalization-group (DMRG) approaches, $\frac{1415}{115}$ however these techniques are so far restricted to lattice problems in one spatial dimension. Correlated magnetism on higher-dimensional lattices starts to be investigated $\frac{16 \mid 17}{16}$ by time-dependent dynamical mean-field theory (TD-DMFT) $\frac{18}{20}$ But the Keldysh-based method is numerically very heavy and not yet capable to address general problems dealing with an interplay between doping, metallicity, non-collinearity, inhomogeneous features and/or multi-orbital degrees of freedom. There are simpler e.g. Gutzwiller-based nonequilibrium schemes ${ }^{[21}$ In view of concrete spin-dynamics problems those however are so far restricted to the linearresponse limit ${ }^{22} 24$ or are put into practise within idealized model settings. 25

The aim of the present work is to introduce a novel approach to the spin dynamics emerging from correlated electrons, without restriction to too advantageous theory limits. Our real-space implementation of the time-dependent rotational-invariant auxiliary (or 'slave')-boson (TD-RISB) scheme opens the possibility to study general interacting problems out of equilibrium in an efficient and flexible way. This is here demonstrated by its reliability in mediating between the linear-response limit of spin excitations within the Slater and the Heisenberg limits of the Hubbard model. Global and local excitations beyond linear response in the doped Mottinsulating regime exhibit the vast potential of the versatile framework.

The RISB approach to equilibrium problems of multiorbital correlated electrons has been proven successful for model Hamiltonians 26 as well as in the context of realistic materials. ${ }^{33134}$ In essence, the method may be characterized on the operator level by the decomposition of the complete electron degree of freedom $c^{(\dagger)}$ into a low-energy quasiparticle (fermionic) part $f^{(\dagger)}$ and high-energy Hubbard (bosonic) representants $\left\{\phi^{(\dagger)}\right\}$. In a key approximation, the bosonic degrees of freedom are treated on the mean-field level, hence the simplified elec- 
tron self-energy is, as in more general DMFT, purely local. Extension to the time domain, motivated by previous Gutzwiller advances, $\stackrel{21}{2}$ opens the possibility for the description of intricate multi-orbital electon correlations out of equilibrium $\frac{3135}{}$ Time propagation of quasiparticle (QP) and bosonic degrees of freedom is described by a set of coupled non-linear Schrödinger-like equations. Here the TD version is implemented in real space, which opens the possibility to study inhomogeneous lattice dynamics as well as two-particle excitations beyond linear response.

\section{MODEL AND METHODOLOGY}

\section{A. Interacting Hamiltonian}

We focus on the one-band Hubbard model on a square lattice with hopping $\tau$, on-site interaction $U$ as well as a space- and time-dependent magnetic field $B(\mathbf{r}, t)$, i.e.

$$
\mathcal{H}(t)=-\sum_{i j \sigma} \tau_{i j} c_{i \sigma}^{\dagger} c_{j \sigma}-\sum_{i} \mathbf{B}_{i}(t) \cdot \mathbf{S}_{i}+U \sum_{i} n_{i \uparrow} n_{i \downarrow}
$$

where $i, j$ label lattice sites, $\sigma=\uparrow, \downarrow$ marks the spin projection and $\mathbf{S}$ is the local spin operator. A real-space lattice of size $N=6 \times 6$ (cf. Fig. 1) with periodic boundary conditions is employed. Extension to multi-orbital problems with a concrete materials background is straightforward 35136

\section{B. Rotational-invariant slave-boson (RISB) representation}

To introduce our framework, lets first discuss the equilibrium case at $t=0$ and remind of the state-of-the-art RISB technique. For more details and generalizations we refer to Ref. 27.

In real space, the one-band limit of the method asks for a set

$$
\text { site } i=1, N: f_{\uparrow}, f_{\downarrow} ; \phi_{E},\left(\begin{array}{cc}
\phi_{\uparrow \uparrow} & \phi_{\uparrow \downarrow} \\
\phi_{\downarrow \uparrow} & \phi_{\downarrow \downarrow}
\end{array}\right), \phi_{D}
$$

of degrees of freedom to provide a complete state representation, whereby $\phi_{E}, \phi_{D}$ are associated with the empty and doubly-occupied site. A full coverage of the spinrotational latitude is given by the slave-boson matrix for the singly-occupied site, and the whole set of bosons still accounts for possible local charge fluctuations. The four possible electron states $\{A\}=\{|E\rangle,|\uparrow\rangle,|\downarrow| D\rangle$,$\} on a$ single lattice site are represented in RISB upon action

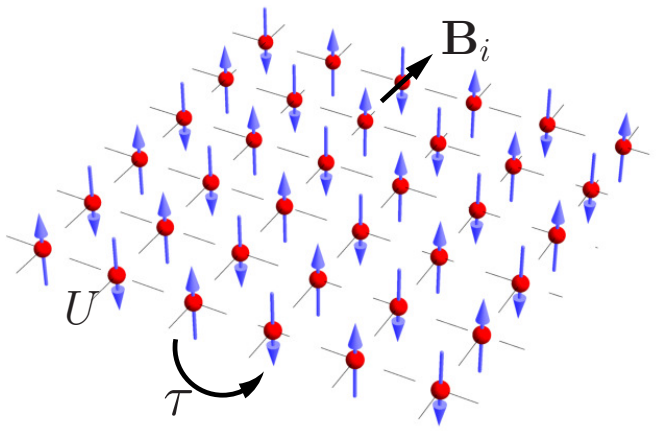

FIG. 1. (color online) Hubbard model with applied sitedependent magnetic field on a $6 \times 6$ real-space lattice.

on the vauum state $\mid$ vac $\rangle$ as follows

$$
\begin{aligned}
|E\rangle=|0\rangle & =\phi_{E}^{\dagger}|\mathrm{vac}\rangle \\
|\uparrow\rangle & =\frac{1}{2}\left\{\phi_{\uparrow \uparrow}^{\dagger} f_{\uparrow}^{\dagger}+\phi_{\uparrow \downarrow} f_{\downarrow}^{\dagger}\right\}|\mathrm{vac}\rangle \\
|\downarrow\rangle & =\frac{1}{2}\left\{\phi_{\downarrow \uparrow}^{\dagger} f_{\uparrow}^{\dagger}+\phi_{\downarrow \downarrow} f_{\downarrow}^{\dagger}\right\}|\mathrm{vac}\rangle \\
|D\rangle=|\uparrow \downarrow\rangle & =\phi_{D}^{\dagger}|\mathrm{vac}\rangle .
\end{aligned}
$$

The second index on the single-particle bosons refer to a QP degree of freedom, whereas the first index generally is associated with the local state. In order to select the true physical states, the constraints

$$
\begin{aligned}
1 & =\phi_{E}^{\dagger} \phi_{E}+\sum_{\sigma \sigma^{\prime}} \phi_{\sigma \sigma^{\prime}}^{\dagger} \phi_{\sigma \sigma^{\prime}}+\phi_{D}^{\dagger} \phi_{D} \\
f_{\sigma}^{\dagger} f_{\sigma} & =\phi_{D}^{\dagger} \phi_{D}+\sum_{\sigma^{\prime}} \phi_{\sigma \sigma^{\prime}}^{\dagger} \phi_{\sigma \sigma^{\prime}} \\
f_{\sigma}^{\dagger} f_{\bar{\sigma}} & =\sum_{\sigma^{\prime}} \phi_{\sigma^{\prime} \bar{\sigma}}^{\dagger} \phi_{\sigma^{\prime} \sigma}
\end{aligned}
$$

have to be enforced on each site. We then write the interacting Hamiltonian (1) in equilibrium as $\underline{\mathcal{H}}=\underline{\mathcal{H}}^{\text {(kin) }}+$ $\sum_{i} \underline{\mathcal{H}}_{i}^{\text {(loc) }}$, whereby the electron operator is expressed through

$$
\begin{aligned}
\underline{c}_{i \sigma}^{\dagger} & =\frac{1}{\sqrt{2}} \sum_{\sigma^{\prime}}\left\{\phi_{i \sigma \sigma^{\prime}}^{\dagger} \phi_{i E}-(-1)^{\delta_{\sigma \sigma^{\prime}}} \phi_{i D}^{\dagger} \phi_{i \bar{\sigma} \bar{\sigma}^{\prime}}\right\} f_{i \sigma^{\prime}}^{\dagger} \\
& \equiv \sum_{\sigma^{\prime}} R_{i \sigma^{\prime} \sigma}^{\dagger} f_{i \sigma^{\prime}}^{\dagger} .
\end{aligned}
$$

Note that as common in slave-particle theories, there is a gauge symmetry providing some freedom in the actual representation of the QP indices on each lattice site. But as shown in Ref. 27, physical observables remain of course gauge invariant. Let us mention that in this regard, Lanata et al ${ }^{37}$ recently proposed an alternative RISB representation.

We can write the kinetic Hamiltonian readily as

$$
\underline{\mathcal{H}}^{(\mathrm{kin})}=\sum_{i j} \sum_{\sigma \sigma^{\prime} \sigma^{\prime \prime}} R_{i \sigma^{\prime} \sigma}^{\dagger} \tau_{i j} R_{j \sigma \sigma^{\prime \prime}} f_{i \sigma^{\prime}}^{\dagger} f_{j \sigma^{\prime \prime}} .
$$


One may define a local QP weight via $\mathbf{Z}_{i}=\mathbf{R}_{i} \mathbf{R}_{i}^{\dagger}$. Eqns. 10 111) already render the key feature for describing non-trivial spin dynamics obvious: the rotationalinvariant framework allows for spin $\sigma, \sigma^{\prime}$ exchange through local-multiplet excitations via QP hopping processes.

To represent the local Hamiltonian, one uses the fact that any local operator $\mathcal{O}$ may be written in quadratic terms of the bosonic degrees of freedom. The general RISB form is given by

$$
\underline{\mathcal{O}}=\sum_{A A^{\prime}}\left\langle A|\mathcal{O}| A^{\prime}\right\rangle \sum_{\gamma} \phi_{A \gamma}^{\dagger} \phi_{A^{\prime} \gamma}
$$

For the local Hubbard interaction, i.e. $\underline{\mathcal{O}}=U \underline{n}_{i \uparrow} \underline{n}_{i \downarrow}$, the slave-boson representation $\mathcal{H}_{\mathrm{U}}=U \phi_{D}^{\dagger} \phi_{D}$ is readily obtained. With the help of the Pauli matrices $\mathcal{S}_{\nu}$ along the component $\nu=x, y, z$, the local spin operator generally reads $\mathbf{S}=\frac{1}{2} \sum_{\sigma \sigma^{\prime}} c_{\sigma}^{\dagger} \overrightarrow{\mathcal{S}} c_{\sigma^{\prime}}$. In the Hamiltonian 11 it appears in the product form $\mathbf{B} \cdot \mathbf{S}=\sum_{\nu} B_{\nu} S_{\nu}$. Hence,

$$
\begin{aligned}
\underline{\mathbf{B} \cdot \mathbf{S}} & =\sum_{A A^{\prime}}\left\langle A|\mathbf{B} \cdot \mathbf{S}| A^{\prime}\right\rangle \sum_{\gamma} \phi_{A \gamma}^{\dagger} \phi_{A^{\prime} \gamma} \\
& =\frac{1}{2} \sum_{\nu} B_{\nu} \sum_{\sigma \sigma^{\prime}}\left\langle\sigma\left|S_{\nu}\right| \sigma^{\prime}\right\rangle \sum_{\sigma^{\prime \prime}} \phi_{\sigma \sigma^{\prime \prime}}^{\dagger} \phi_{\sigma^{\prime} \sigma^{\prime \prime}} \\
& =\frac{1}{2} \sum_{\nu} B_{\nu} \sum_{\sigma \sigma^{\prime}} \mathcal{S}_{\nu \sigma \sigma^{\prime}} \sum_{\sigma^{\prime \prime}} \phi_{\sigma \sigma^{\prime \prime}}^{\dagger} \phi_{\sigma^{\prime} \sigma^{\prime \prime}} .
\end{aligned}
$$

Together with the Hubbard interaction, this completes the local-Hamiltonian representation

$$
\begin{aligned}
\underline{\mathcal{H}}_{i}^{(\mathrm{loc})}= & -\frac{1}{2} \sum_{\nu=x y z} B_{i \nu} \sum_{\sigma \sigma^{\prime} \sigma^{\prime \prime}} \mathcal{S}_{\nu \sigma \sigma^{\prime}} \phi_{i \sigma \sigma^{\prime \prime}}^{\dagger} \phi_{i \sigma^{\prime} \sigma^{\prime \prime}}+ \\
& +U \phi_{i D}^{\dagger} \phi_{i D} .
\end{aligned}
$$

For the rest of the paper, the hopping $\tau$ is restricted to nearest neigbours. Energies(Times) will be given in units of the (inverse) half-bandwidth $\frac{W}{2}=4 \tau$.

In the following, the mean-field limit of the RISB theory is put into practise. Within that limit (i.e. at saddlepoint) the bosonic degrees of freedom are condensed and treated as $c$-numbers. Proper normalizations of the respective RISB electronic operators have the to be invoked to ensure a coherent description in the different interaction limits. This means that the $R$-matrices introduced in eq. (10) are normalized such to yield the correct QPweight limit $Z=1$ at weak coupling. For a detailed discussion of this matter we refer to Ref. 27. Note that it has been shown ${ }^{38}$ that the mean-field RISB method is equivalent to the generalized multi-orbital Gutzwiller approach 39

\section{AFM ground state at half filling}

For our considerations, the initial phase at $t=0$ is defined by the interacting lattice in equilibrium. It is

\begin{tabular}{c|c|c|c|c} 
Lattice size & $U$ & $|\langle m\rangle| \leq 1.0$ & $\left\langle S^{2}\right\rangle \leq 3 / 4$ & charge gap \\
\hline $6 \times 6$ & 0.4 & 0.39 & 0.46 & 0.06 \\
& 1.0 & 0.62 & 0.56 & 0.33 \\
& 2.0 & 0.86 & 0.67 & 2.40 \\
& 3.0 & 0.94 & 0.71 & 3.22 \\
\hline $8 \times 8$ & 0.4 & 0.34 & 0.45 & 0.06
\end{tabular}

TABLE I. Physical quantities extracted from the homogeneous equilibrium AFM state for different Hubbard $U$ : ordered magnetic moment $\langle m\rangle$, local spin moment $\left\langle S^{2}\right\rangle$ and charge gap.

given by the symmetry-broken antiferromagnetic (AFM) ordered phase close to half filling, where the local moments are collinear initialized in $x$-direction. Some key physical quantities of the equilibrium AFM lattice at half filling as described in RISB are given in Tab. I]

At weak coupling with $U=0.4$, a band-magnetic Slater limit is observed, in which the local magnetic response is comparatively small. Increasing $U$ enhances the local and ordered moments, until at $U=3$ the spins are almost fully polarized. The time-independent form of the Hubbard Hamiltonian (1) then starts to approach a Heisenberg-Hamiltonian form which is fully realized at very large $U$. Note that for all values of $U$ a finite charge gap persists, i.e. the equilibrium ground state is an AFM insulator. Since the paramagnetic Mott transition in the present scheme sets in at $U \sim 3.05$, the value of $U=3$ is located in the strongly correlated regime of the Hubbard model.

\section{Time-dependent RISB scheme}

For the dynamic regime, the equilibrium RISB solution sets the stage. We then approximately propagate the equilibrium solution with the TD Hamiltonian (1). The condensed slave bosons $\phi$ become time dependent and a set of non-linear differential equations governs the problem. Conveniently, a multi-index notation is used, whereby a site-dependent quantity $a_{i}$ can be written as $\bar{a}=(i, a)$. Greek letters label the QP degrees of freedom and $A, A^{\prime}$ describe local states. Then the set of differential equations reads $\$ 0 \mid 41$

$$
\begin{aligned}
i \frac{\partial \eta_{\bar{\beta} \bar{\alpha}}}{\partial t} & =\sum_{\bar{\beta}^{\prime}} \underline{\mathcal{H}}_{\bar{\alpha} \bar{\beta}^{\prime}}^{\mathrm{kin})} \eta_{\bar{\beta} \bar{\beta}^{\prime}} \\
i \frac{\partial \phi_{\bar{A} \bar{\gamma}}}{\partial t} & =\sum_{\bar{A}^{\prime}} \underline{\mathcal{H}}_{\bar{A} \bar{A}^{\prime}}^{(\mathrm{loc})} \phi_{\bar{A}^{\prime} \bar{\gamma}}+\sum_{\bar{\alpha}^{\prime}}^{\text {occ }} \sum_{\bar{\alpha} \bar{\beta}} \eta_{\bar{\alpha} \bar{\alpha}^{\prime}}^{\dagger} \frac{\partial \underline{\mathcal{H}}_{\bar{\alpha} \bar{\beta}}^{(\mathrm{kin})}}{\partial \phi_{\bar{A} \bar{\gamma}}^{\dagger}} \eta_{\bar{\alpha}^{\prime} \bar{\beta}}
\end{aligned}
$$

In the first subset 15 of equations, $\bar{\beta}$ runs only over occupied sites and spin projections, while $\bar{\alpha}, \bar{\beta}^{\prime}$ run over all sites and spin projections. The quantity $\eta$ depicts eigenstates of the renormalized kinetic Hamiltonian $\underline{\mathcal{H}}^{(\mathrm{kin})}$ in real space. The restriction to occupied states is indicated in the second equation subset (16). It means that 
in TD-RISB (as in TD-Gutzwiller) the fixed quasiparticle occupations enter the time evolution of the bosonic (or Gutzwiller-projector) degrees of freedom. Still, note that the character of the QP states is time dependent and non-equilibrium charge fluctuations/transfers of the physical electrons are allowed. A numerical solution of eqns. 15 16) is achieved by using an adaptive RungeKutta scheme of sixth-fifth order ${ }^{42}$. In the TD scheme, the constraints (7 9 ) are established at $t=0$ and then remain fulfilled during the time development, i.e. the constraints are integrals of motion.

A specific excitation of the lattice induced by the magnetic-field term in the Hamiltonian (1) is performed to achieve two goals. First, we want to measure the magnetic excitations with a single TD calculation. Second aim is to efficiently scan the parameter space in order to tune the response regime from the linear-response limit to the fully non-equilibrium domain. The linear-response regime is mainly investigated to compare to previous studies and to establish a basic understanding, while the latter provides new insight in fully TD magnetic excitations.

The necessary mean-field normalizations of the RISB operators, governing also the TD equations 15 16), are identical to the ones at equilibrium and are properly included in the calculations. To illustrate the reliability of our time-dependent scheme, we provide in the appendix results for the canonical TD one-band Mott transition after an interaction quench, in perfect agreement with TD-Gutzwiller calculations. ${ }^{21}$

\section{SPIN DYNAMICS}

We first describe in section III A the modeling of the excitation of the lattice system as well as the monitoring of the subsequent time evolution. In the following sections III B III E different concrete applications are addressed and discussed.

\section{A. Pulsed excitation}

Instead of quenching the system, the TD Hamiltonian is applied in a pulsed form (see Fig. 2a). In a quench scenario, there is a sudden switch of the initial equilibrium Hamiltonian $\mathcal{H}_{\text {ini }}=\mathcal{H}(t=0)$ to a final Hamiltonian $\mathcal{H}_{\text {fin }}=\mathcal{H}(t>0)$. On the contrary in the pulsed case, the final-Hamiltonian form $\mathcal{H}_{\text {fin }}=\mathcal{H}\left(0<t<t_{p}\right)$ holds, whereby $t_{p}$ marks the duration of the magnetic pulse. After time $t_{p}$ the Hamiltonian is switched back to $\mathcal{H}_{\text {ini }}$. Note that both, the general time $t$ and the pulse-duration time $t_{p}$ have the identical zero value.

The site-dependent magnetic field $\mathbf{B}(\mathbf{r}, t)=\mathbf{B}_{i}(t)$ in $\mathcal{H}_{\text {fin }}$ is here chosen as random in the $y z$-plane with zero $x$-component (see Fig. 2b). The fixed absolute value is site independent, i.e. $\left|\overrightarrow{\mathbf{B}}_{i}\right|=B$. Since we deal with a finite lattice resolution, it is beneficial to consider only (a)

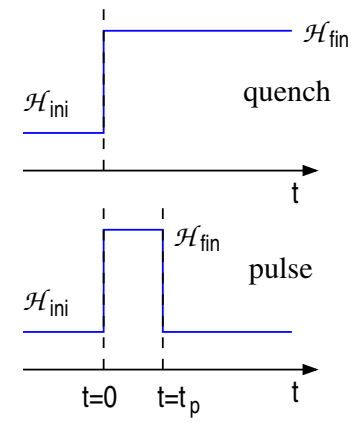

(b)

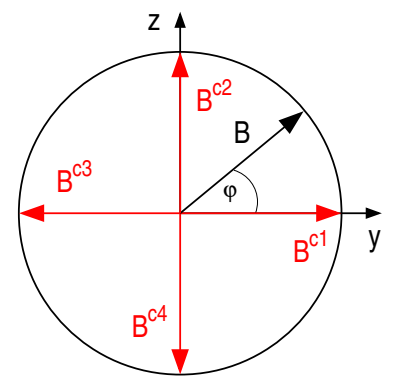

(c)

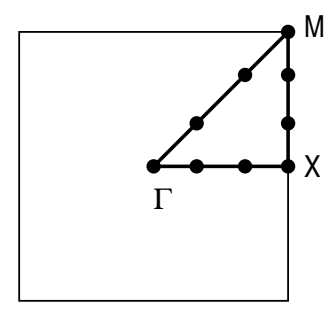

FIG. 2. On the time-dependent calculational settings. (a) Difference between a quench and a pulse with duration time $t_{p}$. (b) Sketch of the possible $C=4$ magnetic-field configurations, applied during $t_{p}$ via $\mathcal{H}_{\text {fin }}$ at lattice site $i$. (d) Brillouin-zone path along the high-symmetry points $\Gamma=(0,0), X=(\pi, 0)$ and $M=(\pi, \pi)$ of the square lattice. Dots mark the accessible points $\mathbf{q}=\left(q_{y}, q_{z}\right)$ for the $6 \times 6$ lattice.

a discrete number of inhomogeneous magnetic-field configurations $\mathbf{B}_{i}$ in the $y z$-plane. We consider the angle $\varphi$ to the $y$-axis and the discrete configurations $\varphi=\frac{2 \pi \nu}{C}$, where $C$ is the total number of configurations and $\nu \in$ $\{1,2, \ldots, C\}$.

Figure $2 \mathrm{~b}$ sketches the possible magnetic-field configurations $\left(\mathbf{B}^{c 1}, \ldots, \mathbf{B}^{c 4}\right)$ for $C=4$ at a given lattice site. In this work, we always choose site-randomly among the $C=4$ configurations. We checked that the results do not depend on this choice. The TD Hamiltonian ensures that all relevant magnetic excitations are generated. Furthermore the linear-response regime is acquired for small $B$ and short $t_{p}$. A strong non-equilibrium regime is quickly obtained for large $B$ and large $t_{p}$. Note that local excitations are easily performed by setting $\mathbf{B}_{i}=0$ for the sites $i$ not to be excited.

To represent the magnetic excitations of the system in reciprocal space, the Fourier transform $\tilde{\mathbf{m}}$ of the TD magnetic moment $\mathbf{m}(\mathbf{r}, t)$ is computed via

$$
\tilde{\mathbf{m}}(\mathbf{q}, \omega)=\frac{1}{(2 \pi)^{3}} \int d \mathbf{r} \int d t \mathbf{m}(\mathbf{r}, t) \mathrm{e}^{-i \mathbf{q} \cdot \mathbf{r}} \mathrm{e}^{-i \omega t},(
$$

where $\mathbf{q}$ marks a point in the two-dimensional reciprocal space. The remaining vector structure of $\tilde{\mathbf{m}}$ enables a separation of longitudinal ( $x$-direction) and transverse ( $y$ - and $z$-direction) modes. The real-space grid limits the resolution in $\mathbf{q}=\left(q_{y}, q_{z}\right)$ and leads to a certain sampling of the Brillouin zone, shown in Fig. 2 f for the $6 \times 6$ lattice. 


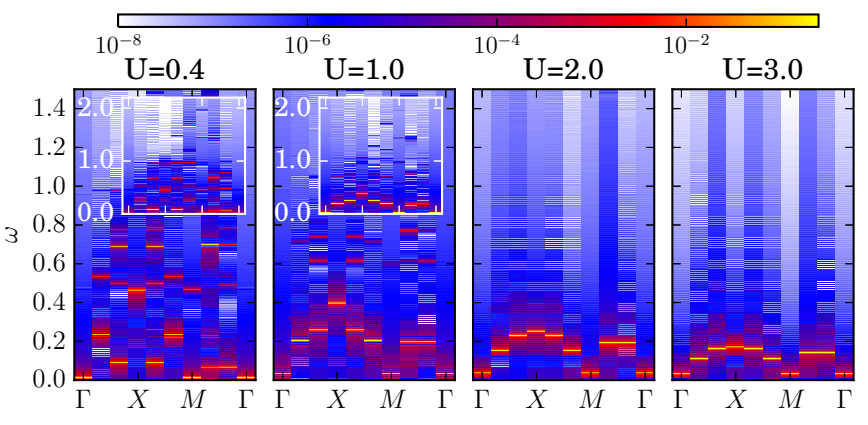

FIG. 3. (color online) $q$-dependent spin-excitation spectra along high-symmetry lines witin the linear-response limit for different interaction strength $U$ at half filling $(B=0.002$, $\left.t_{p}=5\right)$.

Until stated otherwise, the time evolution captures times until a final propagation time $t_{\mathrm{tot}}=1800$. This provides a proper high-energy resolution of the magnetic excitations of the order of $\Delta \omega=0.003$.

\section{B. Slater-to-Heisenberg transition at half filling}

The comparison between spin excitations in the Slater and in the Heisenberg limit of the Hubbard model at half filling serves as a first illustration. For small local interaction strength $U$ the system is close to a degenerate Fermi gas, with nearly absent local-moment physics. But spin polarization may set in via AFM order in reciprocal space through the formation of Slater bands, separated by $U$. Stoner-like excitations amount to inter-band transitions and therefore give rise to a broad continuum of spin excitations, formally starting off at $q=0$ with energy $U$. On the other hand in the large$U$ limit with well-localized electrons, kinetic exchange $\sim t^{2} / U$ leads again to AFM order, now between Heisenberg spins. Spin waves (or magnons) are the low-energy excitations, with well-defined dispersion in $q$-space. Figure 3 documents this Slater-to-Heisenberg transition in the magnetic-excitation spectrum as obtained by realspace TD-RISB within linear response. Though the finite number of $q$-points from the 36 lattice site limits the resolution, the transformation of the broad excitation spectrum for small $U$ to the characteristic AFM magnon dispersion is evident. At $U=2$ this dispersion seems already formed, being further renormalized at $U=3$ to the distinctive width $w=0.2$.

\section{Finite doping at strong coupling}

When introducing holes into the system, the lattice model becomes metallic at smallest doping. In $q$-space, the itinerant background is effective in substantially broadening the $U=3$ magnon spectrum and rendering it quickly incoherent (see Fig. 4a). To measure the

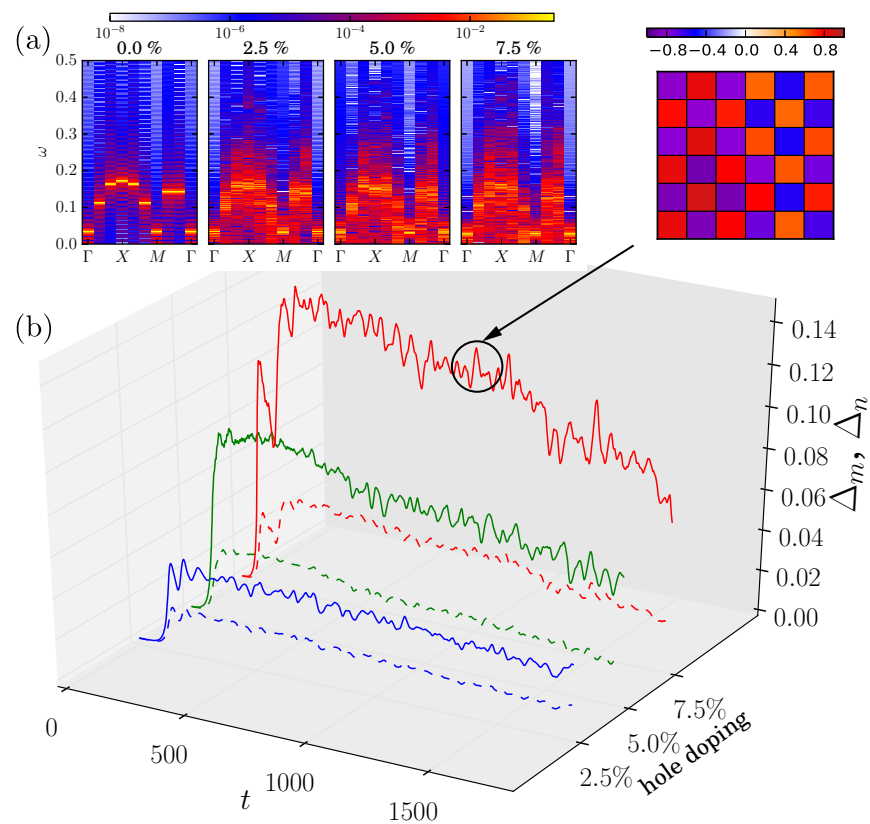

FIG. 4. (color online) Lattice excitation for $U=3$ for different dopings $\left(B=0.002, t_{p}=5\right)$. (a) $q$-dependent spin-excitation spectra along high-symmetry lines. (b) Time evolution of the average inter-site charge (dashed lines) and spin (full lines) differences $\Delta_{m, n}$. At $t=1000$ the site-resolved magnetic moment in real space is depicted.

real-space lattice amplitude fluctuations, it is instructive to define for a given local quantity $Q$ the average TD inter-site difference

$$
\Delta_{Q}(t)=\frac{2}{N(N-1)} \sum_{i, j>i}\left|Q_{i}(t)-Q_{j}(t)\right| .
$$

Figure $4 \mathrm{p}$ displays $\Delta_{Q}(t)$ for the absolute value of the local moment $m=|\mathbf{m}|$ and the local charge $n$, at finite hole doping after the short magnetic pulse $\left(t_{p}=5\right)$. The intersite differences grow with doping, i.e. increasing metallicity leads to stronger inter-site fluctuations, in line with the magnon destruction. As expected, while charge and spin differences seem to act rather coherently, the overall magnitude of $\Delta_{m}(t)$ is much larger. The strong coupling regime suppresses substantial charge fluctuations.

Especially shortly after the pulse, magnetic-moment fluctuations become large and decrease with $t$. Hence time-dependent fluctuations between lattice sites allow for an equilibration of the local observables. Since possible instabilities are encoded in linear-response functions, the fluctuations are not random but are concerted such as to render phase-separating tendencies observable at certain propagation times (see real-space inset in Fig. 4 b). On the other hand, with increasing doping of the strongly correlated AFM lattice, global excitations are truly effective in providing lattice disorder in the time domain. The decrease of the equilibrium AFM order parameter with doping can thus be used to drive a time-dependent phase 
separation into magnetic domains with different sizes of the staggered spin moment. This loss of phase coherency is also part of the reduced magnon lifetime.

\section{Beyond linear response: Magnon destruction and revival}

At half filling, the stability of the strong-coupling magnon dispersion is ensured by the linear-response limit of short pulse time $t_{p}$ and small magnetic-pulse fields. An adiabatic-like description, focussing on the slow dynamics of the stable local moments (formed by the fast dynamics of the electronic degrees of freedom) is sound. However with strong perturbations in magnetic field and pulse time, the degree of non-adiabticity is expected to rise in charge-fluctuating Hubbard systems due to increased incoherent excitation of electrons. In other words, the identification of well-defined slow degrees of freedom and their coherent modes may become difficult. A modeling focus within a sole adiabatic scheme may be too restrictive.

In Fig. 5 we provide results for such a non-linear regime of the dynamic Hubbard lattice model. A sole increase of the absolute value of the pulsed magnetic field, while keeping the pulse time short $\left(t_{p}=5\right)$ does not modify the AFM magnon dispersion by clear means. But an increase of $t_{p}$ results in split-off sidebands to the dispersion away from $\mathbf{q}=\Gamma$. Stimulated by the higher magnetic field, a longer pulse time is effective in significantly reducing
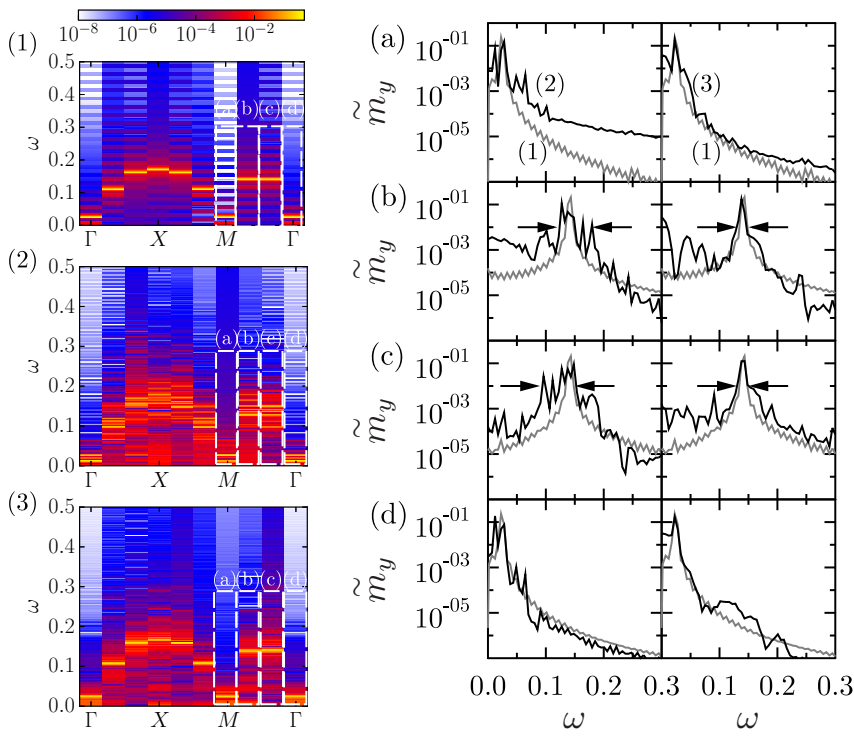

FIG. 5. (color online) $q$-dependent magnetic-excitation spectra $(U=3)$ with increasing TD perturbations at half filling. Left: along high-symmetry lines for increasing pulse time $t_{p}=5,80,320$ in (1), (2) and (3). Right: spectral intensity in the $M-\Gamma$ direction (a-d), as given in the left part of the figure. Arrows indicate the peak sharpening when comparing $t_{p}=80(2)$ with $t_{p}=320$ (3) in this direction within the Brillouin zone. the magnon lifetime. At $t_{p}=80$ the magnon dispersion appears destructed, only to surprisingly recover at $t_{p}=320$, especially inbetween the high-symmetry points $\Gamma$ and $M$. Such physics has already been observed by Zhitomirsky and Chernyshev ${ }^{43}$ who studied magnons in the Heisenberg limit within the self-consistent Born approximation. There the magnons are destroyed above a critical-field strength due to an overlap of the singlemagnon peak with the two-magnon continuum. A further increase of the magnetic field then lead to a reformation of the magnon spectrum. Hence the different energy transfers in our varying pulse times modify the magnonmagnon scattering such that long-lived spin waves become possible again at larger $t_{p}$. The quantitative details of our findings will depend on the size of the simulated time window $t_{\text {tot }}$, but our choice $t_{\text {tot }} \gg t_{p}$ ensures the qualitative result.

\section{E. Beyond linear response: Local excitation in the doped AFM Mott state}

Finally, to show that our method is not bound to global excitations, a local-excitation beyond linear response is studied. We start from a $5 \%$ hole-doped antiferromagnetic state (staggered moment along $x$-direction) at strong coupling and apply an intense and long magnetic pulse $\left(B=0.02, t_{p}=320\right)$ to two AFM-aligned adjacent lattice sites (see Fig. 6a). The pulsed magnetic field is directed along $+z(-z)$ on the first(second) site, to render spin momentum conserved by the excitation process. As shown in Fig. 6b, the $+z$-pulse-excited site switches its magnetic moment shortly after the pulse in that direction. But at the end of the pulse duration the moment points along $(-x, z)$. For longer times, the local moment oscillates mainly in the $x z$-plane. The moment of the second site has a similar time evolution with proper sign changes. It is instructive to display the timeaveraged local quantities, i.e. $\bar{Q}(T)=\frac{1}{T} \int_{0}^{T} d t Q(t)$. Notably for both local moments, time-averaging leads to a loss of a net resulting local magnetization axis (cf. Fig. 6e).

This paramagnetic behavior at long times holds not only for both excited sites, the whole lattice becomes disordered. Figure $6 \mathrm{~d}$ exhibits that the inter-site amplitude fluctuations differ if for $\Delta_{m, n}(t)$ the sum over sites in eq. 18 is chosen as $i, j=$ 'excited,rest' (ER) or $i, j=$ 'rest,rest' (RR). This means, that for ER only the fluctuations between the excited sites and the remaining lattice sites enter $\Delta_{m, n}(t)$, while in the RR mode, fluctuations between all sites but the excited ones are inspected. While the charge fluctations are rather similar for both modes, the RR spin fluctuations are surpisingly stronger and monotonically decaying in time. On the other hand the ER spin fluctuations are smaller below a characterstic time $t_{c} \sim 1000$, i.e. there the excited spins exchange weaker with the other sites. After $t_{c}$ the fluctuations grow and finally even become larger than in the RR sec- 
(a)
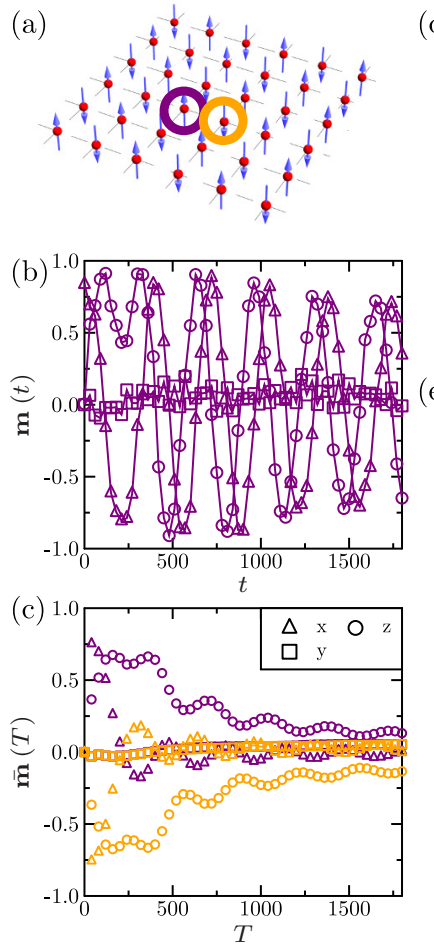

$(\mathrm{d})$

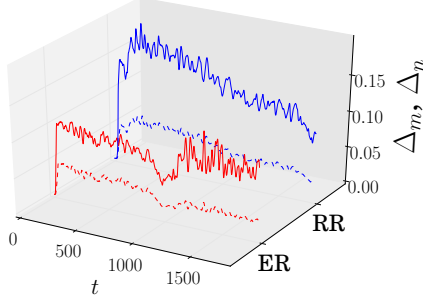

(e)

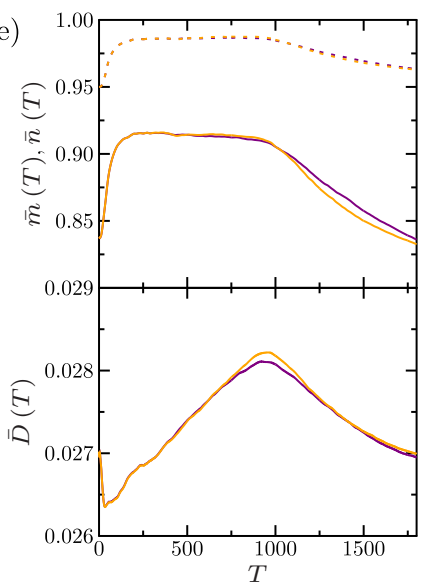

FIG. 6. (color online) Local $\pm B_{z}$ excitation of two lattice sites in the $5 \%$ hole-doped AFM state $(U=3, B=0.02$, $\left.t_{p}=320\right)$. (a) Lattice with excited sites encircled in purple(dark)/orange(grey). In (b),(c) and (e), the data in purple(dark) is associated with the first excited site, data in orange(grey) with the second excited site as depicted in (a). (b) component-resolved TD local magnetic moment $\mathbf{m}$ for one of the excited sites and (c) time-integrated local moment for both excited sites. (d) TD lattice charge and spin fluctuations, resolved via the $\Delta_{Q}$-function (see text). (e) Timeintegrated data on the respective excited sites for (top) the absolute value of the local magnetic moment $m$ and local charge $n$, as well as for (bottom) the local double occupation $D$.

tor. The time $t_{c}$ appears as a pulse-induced coherence time, since during $t_{c}$ the build-up larger local-moment amplitude and local charge on the both excited sites remain nearly constant (see Fig. 6e), forming a plateaulike structure. Only after $t_{c}$ the moment and charge relax to their respective values before the pulse. The time-averaged double occupation $\bar{D}(T)$ increases in the plateau, i.e. the local-correlation strength on the excited sites reduces somewhat below $t_{c}$. Note that the plateau formation results from the high-field pulse and seems not very sensitive to the pulse length $t_{p}$. This model localexcitation scenario documents the principle possibility of inducing/controlling local coherency in doped correlated magnets.

\section{CONCLUSIONS}

We presented a novel general and efficient TD-RISB formulation to deal with the problem of interacting lattice spins within a correlated electron system. The method is suitable in the limit of weak as well as strong correlations and can describe both, itinerant or Mottinsulating environments. Global and/or local excitations are handable, with the resolution of $q$ - as well as $r$ dependent out-of-equilibrium features. We chose some test cases to elucidate the general possibilities of the framework. Of course, the site-random magnetic-field pulse may not be linked to common experimental excitation protocols. It was here mainly utilized to illustrate the method. Implementation of realistic pumpprobe processes is a next natural step. Also treating multi-orbital systems with spin-orbit coupling is highly interesting to resolve TD transfers between angular- and spin-momentum. Coupling to phonons would allow to account for spin-lattice relaxations. An inclusion of explicit inter-site self-energies in cluster extensions is furthermore possible. Finally, the approach is ideally suited to be combined with TD density functional theory to advance on the description of realistic non-equilibrium physics. 44 It could overcome restrictions in the time-domain modeling due to the presently used exchange-correlation functionals.

\section{ACKNOWLEDGMENTS}

We thank C. Ederer and M. Sayad for helpful discussions. This research was supported by the DFGSFB925. M.I.K. acknowledges support from European Research Council (ERC) Advanced Grant No. 338957 FEMTO/NANO. Computations were performed at the University of Hamburg and at the North-German Supercomputing Alliance (HLRN) under Grant No. hhp00026.

\section{Appendix: Dynamic one-band Mott transition}

As a reference, we consider the one-band Hubbard Hamiltonian and provide results for the time-dependent Mott problem after an interaction quench. Our data may be directly compared to the one based on the original TDGutzwiller study of Schiró and Fabrizio. ${ }^{21}$ Here we employ the problem on a simple cubic lattice with nearestneigbor dispersion of bandwidth $W_{\mathrm{sc}}=12 \tau$. Note that for these calculations we do not use a real-space approach, but define the primitive unit cell and solve the problem by utilizing a fine-grid $k$-point mesh. In RISB, the equilibrium Mott transition occurs at $U_{c}=2.65 \frac{W_{\mathrm{sc}}}{2}$. As a natural choice, one rescales the energetics of the non-equilibrium problem in units of the critical equilibrium $U_{c}$. The relevant interaction parameters are then expressed via $u_{\text {ini }}=U_{\text {ini }} / U_{c}$ and $u_{\text {fin }}=U_{\text {fin }} / U_{c} \equiv u$. 

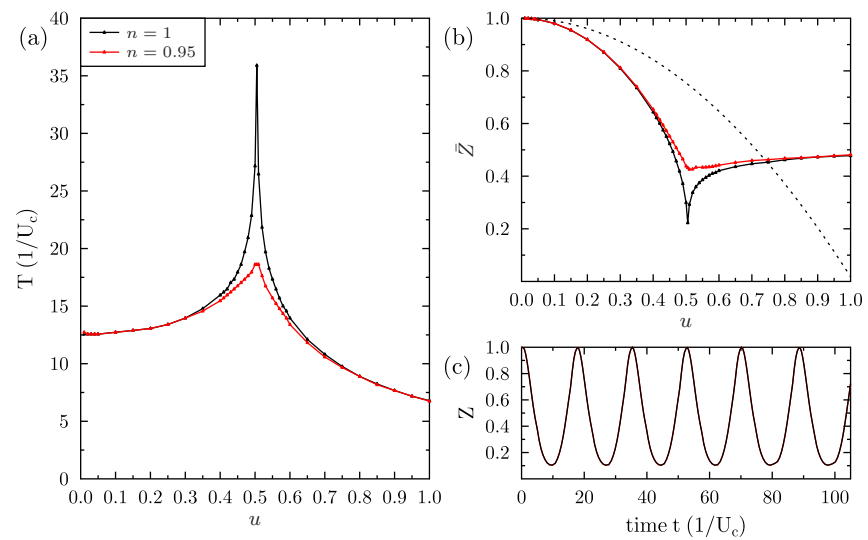

FIG. 7. (color online) TD-RISB data for the one-band dynamical Mott transition on a simple-cubic lattice. (a) Period $T$ of oscillations of the QP weight $Z(t)$ with reduced quenched $u$ at half filling $n=1$ and at hole doping $n=0.95$. (b) Timeaveraged $\bar{Z}$ with $u$ in half-filled and doped case. Dashed line displays the equilibrium $Z$. (c) Time dependent $Z$ for the hole-doped case $n=0.95$ at $u=0.5$. Note that the finite cutoff of the divergences in $(a, b)$ at half filling is an numerical issue.
From Ref. 21, the dynamical critical interaction strength is given by $u_{c}=\left(1+u_{\text {ini }}\right) / 2$. Since TDGutzwiller and mean-field TD-RISB yield equivalent physics, and we here choose to put the inital $u_{\text {ini }}$ to zero, the dynamical Mott transition should occur at $u_{c}=0.5$ in our calculations. Indeed at half filling with this value of $u$, Fig. 7 a displays the characteristic logarithmic divergence of the period $T$ of oscillations in the QP weight $Z$ (for details see Ref. 21). Small hole doping is shown to cut-off this divergence. As shown in Fig. 7b, the time-averaged QP-weight $\bar{Z}=\frac{1}{t} \int_{0}^{t} d t^{\prime} Z\left(t^{\prime}\right)$ has a similar signature as the equilibrium $Z$, but in comparison the dynamical Mott transition takes place at half of the equilibrium interaction strength. Finite doping prohibits a vanishing $\bar{Z}$ and hence the dynamical Mott transition (as in the equilibirum case) remains absent in that case.

For illustration, Fig. 7f exhibits the oscillating $Z(t)$ in the hole-doped scenario. These oscillations are due to the mean-field description of the TD-Gutzwiller and TD-RISB method, which lack relaxation due to quantum fluctuations. However note that relaxation and/or thermalization phenomena may still occur by other means through an increase of the numbers of degrees of freedom in the system under consideration. This was shown in recent TD multi-orbital studies ${ }^{31135}$ and is also effective in the present real-space work.
1 E. Beaurepaire, J.-C. Merle, A. Daunois, and J.-Y. Bigot, Phys. Rev. Lett. 76, 4250 (1996).

2 A. Melnikov, H. Prima-Garcia, M. Lisowski, T. Gießel, R. Weber, R. Schmidt, C. Gahl, N. M. Bulgakova, U. Bovensiepen, and M. Weinelt, Phys. Rev. Lett. 100, 107202 (2008).

3 D. Fausti, R. I. Tobey, N. Dean, S. Kaiser, A. Dienst, M. C. Hoffmann, S. Pyon, T. Takayama, H. Takagi, and A. Cavalleri, Science 331, 189 (2011).

4 F. Schmitt, P. S. Kirchmann, U. Bovensiepen, R. G. Moore, L. Rettig, M. Krenz, J.-H. Chu, N. Ru, L. Perfetti, D. H. Lu, et al., Science 321, 1649 (2008).

5 S. Hellmann, T. Rohwer, M. Kalläne, K. Hanff, C. S. A. Stange, A. Carr, M. M. Murnane, H. C. Kapteyn, L. Kipp, M. Bauer, et al., Nat. Commun. 3, 1069 (2012).

${ }^{6}$ R. J. Elliott, Phys. Rev. 96, 266 (1954).

7 Y. Yafet, in Solid State Physics (Academic, New York, 1963), vol. 14.

8 D. Steiauf and M. Fähnle, Phys. Rev. B 79, 140401 (2009).

9 M. Krauß, T. Roth, S. Alebrand, D. Steil, M. Cinchetti, M. Aeschlimann, and H. C. Schneider, Phys. Rev. B 80, 180407 (2009).

10 T. A. Ostler, J. Barker, R. F. L. Evans, R. W. Chantrell, U. Atxitia, O. Chubykalo-Fesenko, S. E. Moussaoui, L. B. P. J. L. Guyader, E. Mengotti, L. J. Heyderman, et al., Nat. Commun. 3, 666 (2012).

11 A. Secchi, A. I. Lichtenstein, and M. I. Katsnelson, Ann. Phys. 360, 61 (2015).

12 R. Chimata, L. Isaeva, K. Kádas, A. Bergman, B. Sanyal, J. H. Mentink, M. I. Katsnelson, T. Rasing, A. Kirilyuk, A. Kimel, et al., Phys. Rev. B 92, 094411 (2015).
13 M. Sayad and M. Potthoff, New. J. Phys. 17, 113058 (2015).

14 S. R. White and A. E. Feiguin, Phys. Rev. Lett. 93, 076401 (2004).

15 A. J. Daley, C. Kollath, U. Schollwöck, and G. Vidal, J. Stat. Mech. p. 04005 (2004).

16 P. Werner, N. Tsuji, and M. Eckstein, Phys. Rev. B 86, 205101 (2012).

17 J. H. Mentink and M. Eckstein, Phys. Rev. Lett. 113, 057201 (2014).

18 P. Schmidt and H. Monien, arXiv:cond-mat/0202046 (2002).

19 J. K. Freericks, V. M. Turkowski, and V. Zlatić, Phys. Rev. Lett. 97, 266408 (2006).

${ }^{20}$ M. Eckstein, M. Kollar, and P. Werner, Phys. Rev. Lett. 103, 056403 (2009).

21 M. Schiró and M. Fabrizio, Phys. Rev. Lett. 105, 076401 (2010).

22 G. Seibold, F. Becca, P. Rubin, and J. Lorenzana, Phys. Rev. B 69, 155113 (2004).

23 E. v. Oelsen, G. Seibold, and J. Bünemann, Phys. Rev. Lett. 107, 076402 (2011).

24 J. Bünemann, M. Capone, J. Lorenzana, and G. Seibold, New J. Phys. 15, 053050 (2013).

25 M. Sandri and M. Fabrizio, Phys. Rev. B 88, 165113 (2013).

26 T. Li, P. Wölfle, and P. J. Hirschfeld, Phys. Rev. B 40, 6817 (1989).

27 F. Lechermann, A. Georges, G. Kotliar, and O. Parcollet, Phys. Rev. B 76, 155102 (2007).

28 M. Ferrero, P. S. Cornaglia, L. D. Leo, O. Parcollet, 
G. Kotliar, and A. Georges, Europhys. Lett. 85, 57009 (2009).

29 A. Isidori and M. Capone, Phys. Rev. B 80, 115120 (2009).

30 S. Schuwalow, C. Piefke, and F. Lechermann, Phys. Rev. B 85, 205132 (2012).

31 M. Behrmann and F. Lechermann, Phys. Rev. B 91, 075110 (2015).

32 J. I. Facio, V. Vildosola, D. J. García, and P. S. Cornaglia, arXiv:1605.08652 (2016).

33 F. Lechermann, Phys. Rev. Lett. 102, 046403 (2009).

34 I. I. Mazin, H. O. Jeschke, F. Lechermann, H. Lee, M. Fink, R. Thomale, and R. Valenti, Nat. Commun. 5, 4261 (2014).

35 M. Behrmann, M. Fabrizio, and F. Lechermann, Phys. Rev. B 88, 035116 (2013).

36 M. Behrmann and F. Lechermann, Phys. Rev. B 92, 125148 (2015).
37 N. Lanatà, Y. Yao, X. Deng, V. Dobrosaljević, and G. Kotliar, arXiv:1606.09614 (2016).

38 J. Bünemann and F. Gebhard, Phys. Rev. B 76, 193104 (2007).

39 J. Bünemann, W. Weber, and F. Gebhard, Phys. Rev. B 57, 6896 (1998).

40 M. Behrmann, Doctoral Thesis (2016).

41 M. Fabrizio, in New Materials for Thermoelectric Applications: Theory and Experiment (Springer Netherlands, 2013), chap. 16.

42 J. Verner, Numerical Algorithms 53, 383 (2010).

43 M. E. Zhitomirsky and A. L. Chernyshev, Phys. Rev. Lett. 82, 4536 (1999).

44 K. Krieger, J. K. Dewhurst, P. Elliott, S. Sharma, and E. K. U. Gross, J. Chem. Theory Comput. 11, 4870 (2015). 